\title{
RIPARIAN VEGETATION ON THE LEFT TRIBUTARIES OF THE DANUBE ALONG THE “CLISURA” CROSS VALLEY
}

Erika SCHNEIDER-BINDER *

\begin{abstract}
* Karlsruhe Institute for Technology - University of Land Baden-Württemberg and Research Centre of the Helmholtz Society, Institute for Geography and Geoecology, Department WWF-Institute for Floodplain Ecology, Josefstrasse 1, Rastatt, Germany, D-76437, erika.schneider@kit.partner.edu, erika.schb@t-online.de
\end{abstract}

DOI: 10.1515/trser-2015-0091

KEYWORDS: riparian gallery like forests, tall herbaceous fringes, forests of ravines, human impact by dam construction, Romania.

\section{ABSTRACT}

The author presents the riparian vegetation of the left tributaries of the Danube in the "Clisura" area including the habitat types 91E0* Alluvial forests with Alnus glutinosa and Fraxinus excelsior (Alno-Padion, Alnion incanae, Salicion albae), 6430 Hydrophilous tall herb fringe communities and other interlocking habitats. They are analysed from the ecological and the phytocoenological point of view. Discussed are also their phytogeographical particularities. Furthermore are emphasized the changes of the vegetation on the lower part of the tributaries as a consequence of the construction of the Iron Gate power plant and the backwater situation. Finally the conservation status of the riparian habitats is analysed in the context of their importance for the European Natura 2000 network.

ZUSAMMENFASSUNG: Die Ufervegetation der linken Zuflüsse der Donau im Durchbruchtal "Clisura".

Vorliegende Arbeit befasst sich mit den Ufer begleitenden Pflanzengesellschaften, der linken Nebenflüsse der Donau in ihrem "Clisura" genannten Durchbruchtal, die den Habitattypen 91E0* Erlen und Eschenwälder und Weichholzauenwälder an Fließgewässern, 6430 Feuchte Hochstaudensäume der planaren bis alpinen Höhenstufe sowie anderen mit ihnen eng verbundenen Habitattypen zugeordnet sind. Sie werden aus ökologischer und phytocoenologischer Sicht analysiert, wobei auch auf ihre pflanzengeographischen Besonderheiten aufmerksam gemacht wird. Weiterhin wird auf die Veränderungen der hydrologischen Verhältnisse infolge des Staustufenbaus am Eisernen Tor und dessen Einfluss auf die Vegetation hingewiesen. Schließlich wird der Zustand der Uferhabitate aus naturschutzfachlicher Sicht im Kontext ihrer Bedeutung für das europäische Netzwerk Natura 2000 dargestellt.

REZUMAT: Vegetaţia ripariană a tributarilor de stânga în “Clisura” Dunării.

Lucrarea prezintă comunităţile de plante ripariene ale afluenţilor de stânga ai Dunării de-a lungul „Clisurii” incluse în tipurile de habitat 91E0* Păduri aluviale de Alnus glutinosa, Fraxinus excelsior şi de zăvoaie de salcie, 6430 Comunităţi de lizieră cu ierburi înalte şi higriofile, precum şi alte tipuri de habitate cu care se întrepătrund. Comunităţile sunt analizate din punct de vedere ecologic şi fitocenologic cu referiri şi la particularităţile lor fitogeografice. Sunt discutate de asemenea schimbările condiţiilor hidrologice în urma construcţiei barajului de la Porţile de Fier şi consecinţele acesteia asupra vegetaţiei habitatelor din cursul inferior şi a gurilor râurilor tributare Dunării. În sfârşit habitatele ripariene sunt discutate în contextul importanţei lor pentru rețeaua Europeană Natura 2000. 


\section{INTRODUCTION}

Given that on the "Clisura" stretch of the Danube, i.e. the canon like cross valley through the Carpathians, the cliffs with corresponding vegetation reached till the riverbank, riparian vegetation was less represented. However the Danube tributaries are accompanied by riparian vegetation with changing characteristics from the upstream to the downstream stretches of their courses in function of the geomorphologic structure of the valley.

In its natural state, before the construction of the Iron Gate power plant and the water storage lake, riparian vegetation existed on the Danube upstream the gorge stretch in small floodplains on alluvial deposits and alluvial soils from downstream Baziaş to Moldova Veche (including the Moldova Veche Island) and Pescari, from Cruşeviţa, including Cameniţa and Oreviţa, to Berzasca and Drencova, downstream Plavişeviţa to Orşova and further to Vârciorova (Posea et al., 1972; Glăvan and Geanana, 1972). The larger area where floodplains with typical vegetation existed before 1972, are well delimited by the depressions presented on the map of the Carpathian valley of the Danube by Iancu (1972). They are emerging from Belobreşca to Pescari, Cruşeviţa to Berzasca, a small one around Sviniţa and as well around Dubova, and from Ogradena to Orşova, continuing to the Bahna Valley. The higher area on the level of hardwood or transition forests from soft- to hardwood forests were almost used for small scale agriculture, the riparian vegetation being reduced by man and remained only as gallery-like forest belts. These belts are bordered by tall herbaceous vegetation and in some places covered with curtains of Wilde grape (Vitis sylvestris) (Meusel and Niedermaier, 1985). As typical softwood forest on the Danube in the Iron Gate area is mentioned the association Salici-Populetum (Tx. 1931) Mejer-Drees 1936 (Morariu and Danciu, 1970). These authors mention also the expansion of Amorpha fruticosa which changed the local phytocoenoses.

The secondary herbaceous vegetation replacing the natural communities were - apart from the arable lands - grasslands of Common chouch (Elymus repens), Creeping bentgrass (Agrostis stolonifera), Field meadows foxtail (Alopecurus pratensis) and on the terraces predominantly Golden bluestem (Chrysopogon gryllus) (Doniţă and Roman, 1976). Before flooding the depression of Dubova downstream the gorge of Cazanele Mici were covered by wet grasslands and wetlands interlocking with Willow stands of White and Crick willow (Salix alba, S. fragilis), White poplar (Populus alba), and with sedge (Carex) species. Representative were the abundance of Summer snow flake (Leucojum aestivum), typical for floodplain forests of the association Leucojo-Fraxinetum, and transition stages from soft- to hardwood forests.

Due to the backwater situation of the Danube as a consequence of the dam construction, the small area of riparian vegetation along the Danube River almost disappeared. Riparian gallery like vegetation remained only on the middle and lower streches of the tributaries. But on the lasts they were and are also supposed to many changes, due to the lack of hydrological dynamic on the mouth of tributaries. Taking into account these facts, the objective of the present study is to analyse the riparian habitats with their typical vegetation included in the habitat types 91E0* Alluvial forests with Alnus glutinosa and Fraxinus excelsior (Alno-Padion, Alnion incanae, Salicion albae), 6430 Hidrophilous tall herb fringe communities of plains and of the montane to alpine levels, and also the habitat type 9180* Tilio-Acerion forest of slopes, screes and ravines strongly interlocked with the other two mentioned habitats. An other objective is to highlight the phytogeographical particularities of riparian vegetation of the tributaries in the Clisura area and as well to state changes after the construction of the "Iron Gates" Dam and their consequences, and finally to analyse the conservation status of the riparian habitats with importance for the Natura 2000 network. 


\section{MATERIAL AND METHODS}

During the vegetation period of the year 2014 field researches concerning the riparian habitats were realised on the left tributaries of the "Clisura", the Danube gorge break valley. For comparison have been used also data from previous field activities. Samples were taken according to the method of Braun-Blanquet with the seven degree abundance-dominance scale (Braun-Blanquet, 1964; Borza and Boşcaiu, 1965). Considered were as well aspects concerning the structure of the habitats in strong relation with the water dynamics of the tributaries, the grain size of sediments and the succession of the vegetation along ecological gradients from the river banks to the higher elevations of the river valleys. From upstream to downstream the following valleys have been studied: Ribişel Valley (1), Radimna Valley (2), Boşneag-Valea Mare with Valea Rea and Valea Găurii (3), Liborajdea (= Polevinii) Valley (4), Berzasca Valley with Toroniţa (5), Şirinia Valley near Cozla (6), Liubotina Valley (7), Plavişeviţa Valley (8), Ponicova/râul Morii near Cazanele Mari and Valea Satului (Dubova) (9), Mraconia Valley (10), Ogradena Valley (11) and Eşelniţa Valley (12).

The taken samples are included in phyto-coenological tables and grouped according to characteristic species of the different phytocoenological units or according to ecological gradients and presented as well in the context of the European Union habitats (EUR28, 2013; Gafta and Mountford, 2008). A synthetic table including the phytocoenoses of Beech, Ash, Sycamore (C1), of Ash and Black alder (C2), of Black alder (C3) and of Willow species (C4), represents the associations with their constancy classes as follows $\mathrm{I}=1-20 \%$, II $=21-40 \%$, $\mathrm{III}=41-60, \mathrm{IV}=61-80, \mathrm{~V}=81-100 \%$. The indicator values for wetness $(\mathrm{W})$ and nitrogen $(\mathrm{N})$ are included as well according to Ellenberg et al. (2001). The nomenclature of species is used according to Sârbu et al. (2013) and Ciocârlan (2009).

\section{RESULTS AND DISCUSSION}

The tributaries of the Danube present a large diversity according to the size of their sub-basin and the hydro-morphodynamics, the flow velocity depending on the slope and the discharge of the river. In dependence of these factors, the riparian vegetation of the tributaries on the "Clisura" stretch of the Danube is represented by different plant communities. They are gallery like distributed along the river courses from upstream to downstream.

The structure of the phytocoenoses, according to different layers from the herbaceous to the shrubs and trees, as well their species composition, depends on varying site conditions like the size of the basin of each of the tributaries, the geomorphologic structure of the valley from the springs to the mouth into the Danube (deep incision, width of the valley, etc.), the rocky underground and soil conditions, the length of the river, its flow velocity, discharge and dynamics and as well the changes of the natural conditions by men's intervention, like hydraulic measures. On all the studied tributaries the river banks have a natural morphological structure and the rivers a natural or near-natural hydro-morphological dynamics with erosion and deposition processes. The river dynamics can be observed on the upper and the middle stretch of the streams and rivers and constitutes the base for the structure of the vegetation on the river banks (Fig. 1).

Following the river from upstream to downstream in areas where the river valley is widening, the riparian habitats and their vegetation presents different aspects. The succession along the valley is from stands of beech (Fagus sylvatica), to those of beech and Common ash (Fraxinus excelsior), ash and Black alder (Alnus glutinosa), Black alder as most abundant and dominant species of the tree layer, Black alder and Willow species, in particular Brittle willow (Salix fragilis) and finally to White willow (Salix alba) stands (Tab. 1). 
In general the typical riparian vegetation is poorly represented in the deep incised valleys. On these stretches forests characteristic for narrow ravine like valleys with steep slopes - included in the habitat type 9180* Tilio-Acerion forests of slopes, screes and ravines - reach to the border of the water courses. In that area, where the beech (Fagus sylvatica) is the most abundant and dominant species, can be found isolated trees or small groups of Common ash (Fraxinus excelsior), Sycamore (Acer pseudoplatanus), Elm (Ulmus montana) and some other species (Doniţă et al., 2005, 2006). As well can be found fragments of phytocoenoses of mountainous tall herbaceous vegetation edified by Petasites hybridus and Telekia speciosa, located on the river banks and on stony islets in the river bed between river anastomoses. These tall herbaceous fragmentary fringes are included in the habitat type 6430 Hydrophilous tall herb fringe communities of plains and of the mountainous to alpine levels. Remarkable is that Telekia speciosa, a typical mountainous species of the beech forest level, edifies together with other tall herbaceous species, characteristic fringes. Such fragments of fringes have been observed in the Mraconia Valley on the lowest site of Carpathians at $50 \mathrm{~m}$ height above a.s.l. and in Berzasca Valley at $83 \mathrm{~m}$ a.s.l.

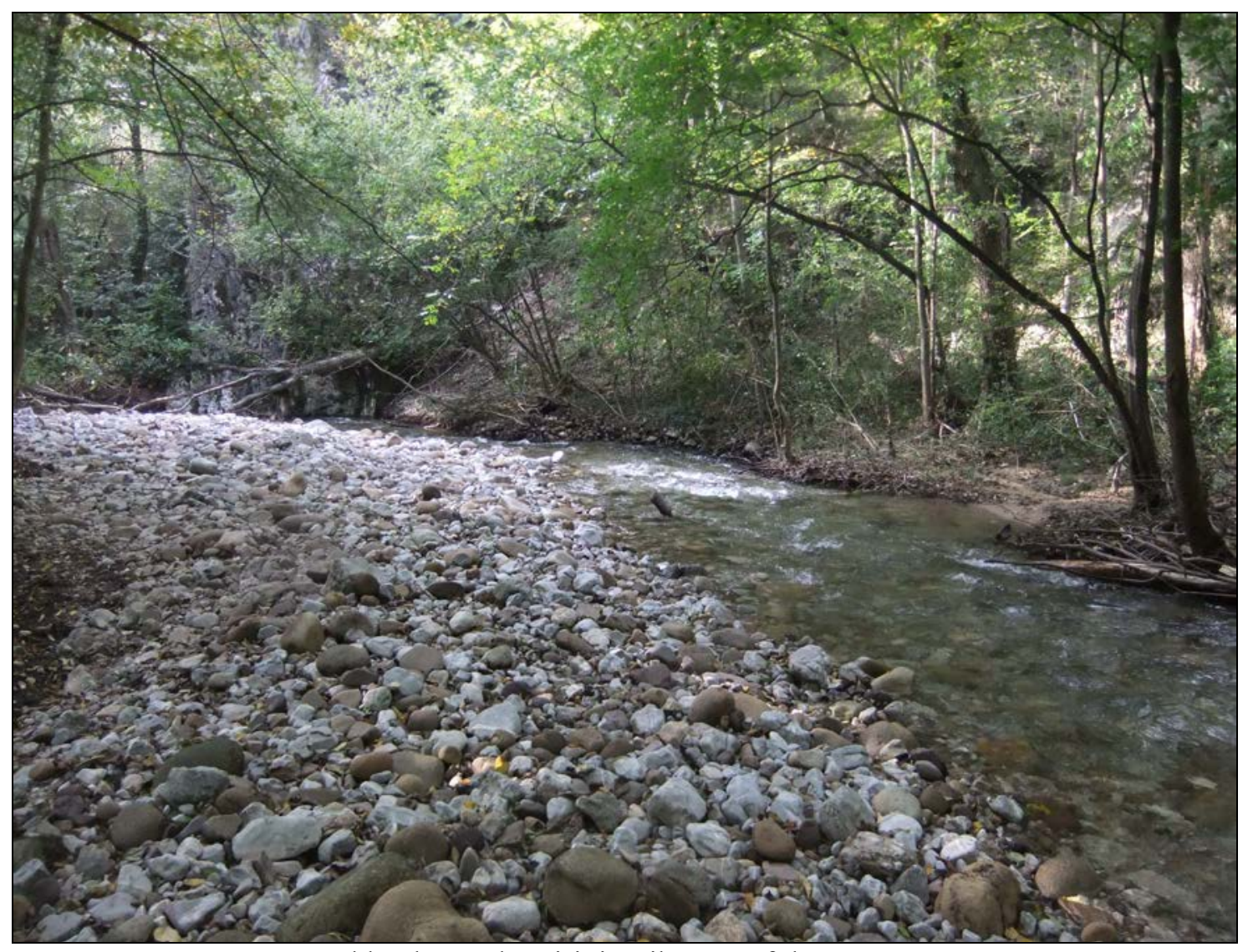

Figure 1: Gravel banks on the Şirinia tributary of the Danube (near Cozla) after high rainfalls and floods in summer 2014. 
Table 1: Synthesis of the riparian phytocoenoses of the Clisura tributaries; Column C1 phytocoenoses of Beech, Ash, Sycamore; C2 phytocoenoses of Ash and Black alder; C3 phytocoenoses of Black alder; C4 phytocoenoses of willow species.

\begin{tabular}{|c|c|c|c|c|c|c|}
\hline & & Number of column & C1 & $\mathrm{C} 2$ & C3 & $\mathrm{C} 4$ \\
\hline & & Number of samples & 11 & 7 & 7 & 7 \\
\hline & & Frequency class & $\mathrm{K}$ & $\mathrm{K}$ & $\mathrm{K}$ & $\mathrm{K}$ \\
\hline $\mathrm{W}$ & $\mathrm{N}$ & Tree layer & & & & \\
\hline $\mathrm{x}$ & 7 & Fraxinus excelsior & $\mathbf{V}$ & $\mathbf{V}$ & - & - \\
\hline 5 & $\mathrm{x}$ & Fagus sylvatica & III & $\mathrm{I}$ & - & - \\
\hline 6 & 7 & Acer pseudoplatanus & III & - & - & - \\
\hline 6 & 7 & Ulmus glabra & III & III & III & III \\
\hline 5 & 6 & Acer campestre & IV & II & III & I \\
\hline $9=$ & $\mathrm{x}$ & Alnus glutinosa & I & $\mathbf{V}$ & $\mathbf{V}$ & II \\
\hline $8=$ & 6 & Prunus padus & II & II & - & - \\
\hline $\mathrm{x}$ & $\mathrm{x}$ & Carpinus betulus & II & II & II & - \\
\hline 5 & 5 & Cerasus avium & $\mathrm{I}$ & II & - & I \\
\hline \multirow[t]{2}{*}{6} & 7 & Juglans regia & I & III & III & II \\
\hline & & Carpinus orientalis & - & - & II & I \\
\hline 4 & 8 & Robinia pseudoacacia & I & - & I & II \\
\hline 5 & 8 & Ailanthus altissima & I & I & - & I \\
\hline $8=$ & 6 & Salix fragilis & II & I & I & III \\
\hline \multirow[t]{2}{*}{$8=$} & 7 & Salix alba & $\mathrm{I}$ & - & I & IV \\
\hline & & Morus alba & I & I & I & III \\
\hline $8=$ & 7 & Populus nigra & & - & - & III \\
\hline $7 \sim$ & 6 & Populus alba & & - & - & I \\
\hline $\mathrm{x}$ & 5 & Corylus avellana & IV & $\mathrm{V}$ & IV & II \\
\hline 5 & $\mathrm{x}$ & Cornus sanguinea & II & II & II & II \\
\hline 5 & 9 & Sambucus nigra & II & $\mathrm{I}$ & III & III \\
\hline 4 & 4 & Crataegus monogyna & $\mathrm{I}$ & III & II & I \\
\hline 5 & 5 & Evonymus europaea & III & III & I & - \\
\hline $\mathrm{x}$ & 7 & Rubus caesius & III & $\mathrm{V}$ & $\mathrm{V}$ & $\mathrm{V}$ \\
\hline 4 & 4 & Cornus mas & I & $\mathrm{I}$ & I & - \\
\hline $9=$ & $\mathrm{x}$ & Alnus glutinosa & - & $\mathrm{I}$ & II & - \\
\hline 4 & $\mathrm{x}$ & Rosa canina & - & - & I & III \\
\hline $8=$ & 6 & Salix fragilis & - & $\mathrm{I}$ & I & I \\
\hline$x=$ & $\mathrm{x}$ & Salix purpurea & - & - & I & $\mathrm{V}$ \\
\hline $8=$ & 5 & Salix triandra & - & - & I & II \\
\hline
\end{tabular}


Table 1 (continued): Synthesis of the riparian phytocoenoses of the Clisura tributaries; Column C1 phytocoenoses of Beech, Ash, Sycamore; C2 phytocoenoses of Ash and Black alder; C3 phytocoenoses of Black alder; C4 phytocoenoses of Willow species.

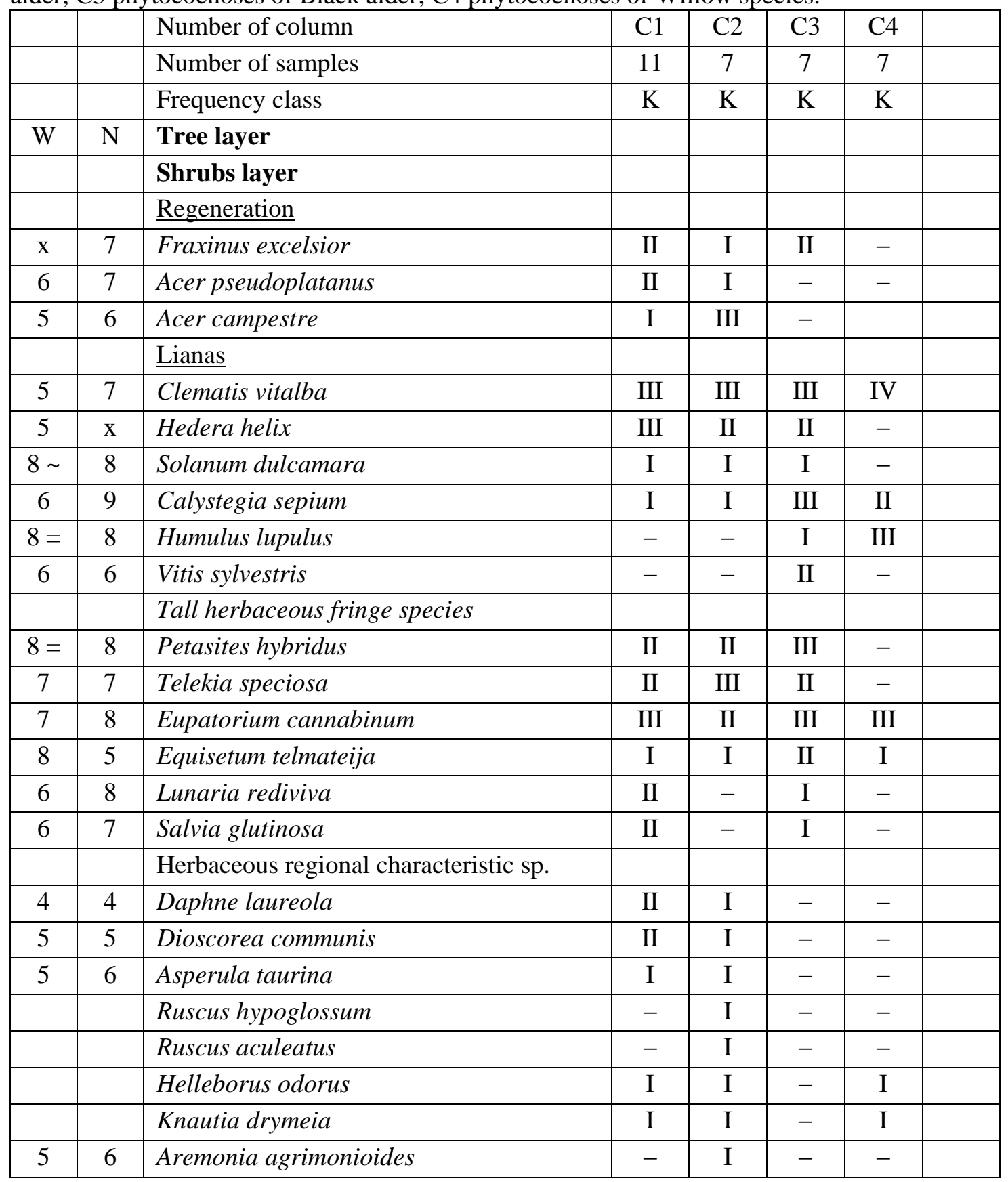


Table 1 (continued): Synthesis of the riparian phytocoenoses of the "Clisura" tributaries; Column C1 phytocoenoses of Beech, Ash, Sycamore; C2 phytocoenoses of Ash and Black alder; C3 phytocoenoses of Black alder; C4 phytocoenoses of willow species.

\begin{tabular}{|c|c|c|c|c|c|c|c|}
\hline & & Number of column & C1 & $\mathrm{C} 2$ & $\mathrm{C} 3$ & $\mathrm{C} 4$ & \\
\hline & & Number of samples & 11 & 7 & 7 & 7 & \\
\hline & & Frequency class & $\mathrm{K}$ & $\mathrm{K}$ & $\mathrm{K}$ & K & \\
\hline & & Shrubs layer & & & & & \\
\hline & & Other heraceous layer species & & & & & \\
\hline 5 & 7 & Parietaria officinalis & III & $\mathrm{V}$ & $\mathrm{V}$ & II & \\
\hline $8=$ & 8 & Polygonum hydropiper & III & IV & IV & III & \\
\hline 6 & 8 & Urtica dioica & IV & III & $\mathrm{V}$ & III & \\
\hline 6 & 8 & Aegopodium podagraria & II & III & III & II & \\
\hline 6 & 8 & Lamium maculatum & III & III & III & $\mathrm{I}$ & \\
\hline 6 & 9 & Rumex obtusifolius & $\mathrm{I}$ & III & II & $\mathrm{I}$ & \\
\hline $9=$ & 8 & Bidens tripartita & $\mathrm{I}$ & II & II & III & \\
\hline 8 & 8 & Symphytum officinale & $\mathrm{I}$ & $\mathrm{I}$ & I & $\mathrm{I}$ & \\
\hline $8=$ & 8 & Myosoton aquaticum & $\mathrm{I}$ & - & III & III & \\
\hline 5 & 8 & Chelidonium majus & II & II & - & $\mathrm{I}$ & \\
\hline $8=$ & 7 & Mentha longifolia & $\mathrm{I}$ & - & $\mathrm{I}$ & $\mathrm{I}$ & \\
\hline 6 & 7 & Glecoma hederacea & II & IV & III & II & \\
\hline 7 & 7 & Stachys sylvatica & $\mathrm{I}$ & III & III & $\mathrm{I}$ & \\
\hline \multirow[t]{2}{*}{$7 \sim$} & $\mathrm{x}$ & Ranunculus repens & II & III & II & III & \\
\hline & & Other herbaceous layer species & & & & & \\
\hline $\mathrm{x} \sim$ & 7 & Elymus repens & $\mathrm{I}$ & I & I & II & \\
\hline $9=$ & 7 & Lycopus europaeus & - & III & $\mathrm{I}$ & $\mathrm{I}$ & \\
\hline $9=$ & 7 & Scrophularia alata & - & - & I & II & \\
\hline 8 & $\mathrm{x}$ & Carex remota & $\mathrm{I}$ & III & II & - & \\
\hline $6 \sim$ & 7 & Dipsacus fullonum & $\mathrm{I}$ & III & I & - & \\
\hline 6 & 7 & Circaea lutetiana & $\mathrm{I}$ & I & - & $\mathrm{I}$ & \\
\hline 5 & 7 & Geum urbanum & $\mathrm{I}$ & III & III & - & \\
\hline 5 & 6 & Mycelis muralis & III & IV & III & $\mathrm{I}$ & \\
\hline 5 & 6 & Dryopteris filix mas & III & I & II & $\mathrm{I}$ & \\
\hline 5 & 6 & Galeopsis tetrahit & $\mathrm{I}$ & II & II & $\mathrm{I}$ & \\
\hline $6 \sim$ & $\mathrm{x}$ & Lysimachia nummularia & $\mathrm{I}$ & I & I & $\mathrm{I}$ & \\
\hline 7 & 6 & Athyrium filix femina & $\mathrm{I}$ & III & $\mathrm{I}$ & - & \\
\hline 7 & 4 & Juncus effusus & - & II & I & $\mathrm{I}$ & \\
\hline 5 & 5 & Stellaria holostea & $\mathrm{I}$ & - & II & II & \\
\hline 7 & 6 & Festuca gigantea & II & III & III & - & \\
\hline
\end{tabular}


Table 1 (continued): Synthesis of the riparian phytocoenoses of the "Clisura" tributaries; Column C1 phytocoenoses of Beech, Ash, Sycamore; C2 phytocoenoses of Ash and Black alder; C3 phytocoenoses of Black alder; C4 phytocoenoses of willow species.

\begin{tabular}{|c|c|c|c|c|c|c|c|}
\hline & & Number of column & C1 & $\mathrm{C} 2$ & $\mathrm{C} 3$ & $\mathrm{C} 4$ & \\
\hline & & Number of samples & 11 & 7 & 7 & 7 & \\
\hline & & Frequency class & $\mathrm{K}$ & $\mathrm{K}$ & $\mathrm{K}$ & K & \\
\hline 5 & 6 & Brachypodium sylvaticum & II & III & III & I & \\
\hline 5 & 6 & Asarum europaeum & II & IV & I & - & \\
\hline 5 & 6 & Pulmonaria officinalis maculosa & III & II & I & - & \\
\hline 7 & 6 & Viola reichenbachiana & II & II & - & - & \\
\hline $\mathrm{x}$ & 7 & Geranium robertianum & III & I & - & - & \\
\hline 3 & 2 & Scabiosa ochroleuca & $\mathrm{I}$ & - & I & $\mathrm{I}$ & \\
\hline 4 & 3 & Coronilla varia & - & I & I & $\mathrm{I}$ & \\
\hline 4 & 3 & Calamintha vulgaris & - & I & II & $\mathrm{I}$ & \\
\hline 11 & 4 & Polygonum amphibium & - & - & - & II & \\
\hline $\mathrm{x}=$ & 5 & Agrostis stolonifera & $\mathrm{I}$ & I & I & III & \\
\hline 4 & 5 & Cichorium intybus & I & I & - & III & \\
\hline 4 & 5 & Achillea millefolium & $\mathrm{I}$ & I & - & $\mathrm{I}$ & \\
\hline 6 & 6 & Ajuga reptans & II & - & II & - & \\
\hline 5 & 5 & Dactylis polygama & $\mathrm{I}$ & - & - & II & \\
\hline $8 \sim$ & $\mathrm{x}$ & Lythrum salicaria & - & - & I & $\mathrm{I}$ & \\
\hline 8 & 4 & Scirpus sylvaticus & - & - & III & - & \\
\hline \multirow[t]{2}{*}{$\mathrm{X}$} & 8 & Stellaria media & - & - & III & - & \\
\hline & & Other accompaining species & & & & & \\
\hline- & - & Phytolacca americana & I & II & I & II & \\
\hline 5 & 8 & Arctium minus & - & II & I & $\mathrm{I}$ & \\
\hline 5 & 8 & Solanum nigrum & - & III & I & $\mathrm{I}$ & \\
\hline 6 & 8 & Erigeron annuus & I & III & - & II & \\
\hline 4 & 5 & Conyza canadensis & $\mathrm{I}$ & II & - & III & \\
\hline 5 & 6 & Plantago major & $\mathrm{I}$ & I & II & - & \\
\hline 4 & 5 & Linaria vulgaris & $\mathrm{I}$ & - & I & III & \\
\hline 5 & 7 & Verbena officinalis & - & I & - & III & \\
\hline 6 & 7 & Physalis alkegengi & $\mathrm{I}$ & I & - & $\mathrm{I}$ & \\
\hline 6 & 8 & Artemisia vulgaris & I & - & I & I & \\
\hline 4 & 8 & Artemisia absinthium & I & - & - & II & \\
\hline $6 \sim$ & $\mathrm{x}$ & Tussilago farfara & $\mathrm{I}$ & II & - & - & \\
\hline 5 & 8 & Galeopsis speciosa & III & - & - & - & \\
\hline 4 & 6 & Artemisia annua & - & - & I & III & \\
\hline 4 & 7 & Setaria viridis & - & - & I & II & \\
\hline
\end{tabular}


Species only in one column with constancy I (1-20\%)

C1: Fagus sylvatica regeneration, Poa nemoralis, Hypericum tetrapterum, Vincetoxicum officinale, Gentiana asclepiadea, Lapsana communis, Luzula sylvatica, Carex elata, Phyllitis scolopendrium, Syringa vulgaris, Euphorbia amygdaloides, Fragaria vesca, Oxalis acetosella, Lathyrus niger, Alliaria petiolate, Dentaria bulbifera, Senecio nemorensis, Angelica sylvestris; C2: Castanea sativa, Fraxinus ornus, Quercus cerris, Poa annua; C3: Alnus glutinosa regeneration, Ailanthus altissima regeneration, Salix viminalis, Berteroa incana, Potentilla tormentilla, Saponaria officinalis, Salix cinerea, Viola mirabilis, Marchantia polymoprpha, Rumex crispus, Stachys palustris, Mentha aquatic, Taraxacum officinale, Anthriscus sylvestris; C4: Populus canescens, Amorpha fruticose, Salix caprea, Echinocystis echinata, Aster lanceolatus, Anchusa officinalis, Melandrium album, Pulicaria vulgaris, Veronica beccabunga, Coronilla emerus, Melilotus albus, Coronilla varia, Cirsium vulgare, Galega officinalis, Portulaca oleracea, Cynodon dactylon, Galium album, Convolvulus arvensis, Eragrostis minor, Silene vulgaris.

Species in two columns with constancy I (1-20\%): C1, C2: Galium schultesii; C1, C3: Ambrosia artemisiifolia, Asplenium trichomanes, Pyrus pyraster (tree layer), Trifolium repens; C1, C4: Potentilla reptans, Robinia pseudoacacia (regen.), Sambucus ebulus; C2, C3: Echinocloa crus-gallii, Fraxinus excelsior, Galinsoga parviflora, Morus alba, Physocaulis nodosus, Robinia pseudoacacia; C3, C4: Astragalus glycyphyllos, Centaurea biebersteinii, Cynodon dactylon, Lythrum salicaria, Myrrhoides nodosa, Xanthium strumarium. Species in one column with constancy II (21-40\%) in one column: C1: Geranium macrorhizum, Prunella vulgaris, Sedum telephium ssp. maximum; C3: Veronica chamaedrys; C4: Chenopodium botrys, Pulicaria dysenterica.

Phytocoenoses of Common ash (Fraxinus excelsior) and Black alder (Alnus glutinosa) and as well those edified by White and Brittle willow and other willow species - including as well all transition stages - take part of the habitat type 91E0*Alluvial forests with Alnus glutinosa and Fraxinus excelsior (Alno-Padion, Alnion incanae, Salicion albae).

Between 450 and $200 \mathrm{~m}$ height a.s.l. the Black alder (Alnus glutinosa) is in general poorly represented, but downstream at the height of $200 \mathrm{~m}$ it is more apparent edifying together with the ash characteristic phytocoenoses, with high abundance-dominance values, its optimum being clear visible (Tab. 2).

Phytocoenoses of Black alder have been described from the Clisura area i.e. the Radimna Valley by Coste 1975 as association Stellario-Alnetum glutinosae (Kästner 1938) Lohm. 1957 (Coste 1984). Remarkable in the phytocoenoses is the high number of nitrophilous species with indicator values 7, 8, 9 (Ellenberg et al., 2001) such are Parietaria officinalis, Glecoma hederacea, Polygonum hydropiper, Urtica dioica, Aegopodium podagraria, Lamium maculatum, Rumex obtusifolius and many others (Tabs. 1 and 2). The high number and abundance-dominance of nitrophilous species indicate a similarity to the association Aegopodio-Alnetum glutinosae Kárpáti and Jurko 1964, described from the Crişul Repede Gorge valley (Sanda et al., 2008).

The observations concerning high number of nitrophilous species, i.e. eutrophication, corresponds to those of the Lower Danube Cama Dinu area, upstream Giurgiu (Mölder and Schneider-Binder, 2011). 
Table 2: Phytocoenoses with Black Alder (Alnus glutinosa) and Common Ash (Fraxinus excelsior) in the middle and lower stretches of the tributaries ( 2 = Radimna, 4 = Liborajdea, 5 = Berzasca, $6=$ Şirinea, $7=$ Liubotina, $10=$ Mraconia, $11=$ Ogradena, 12 = Eşelniţa).

\begin{tabular}{|c|c|c|c|c|c|c|c|c|c|c|c|c|c|c|}
\hline No. of column & 1 & 2 & 3 & 4 & 5 & 6 & & 7 & 8 & 9 & 10 & 11 & 12 & \\
\hline No. of river valley & 12 & 2 & 12 & 11 & 6 & 7 & & 4 & 5 & 5 & 7 & 10 & 11 & \\
\hline No. of field sample & 3 & 19 & 4 & 27 & 10 & 9 & $\mathrm{~F}$ & 22 & 24 & 23 & 8 & 6 & 26 & $\mathrm{~F}$ \\
\hline m above a.s.l. & 194 & 130 & 127 & 112 & 91 & 85 & & 103 & 104 & 83 & 104 & 50 & 169 & \\
\hline \multicolumn{15}{|l|}{ Trees layer } \\
\hline Alnus glutinosa & 4 & 3 & 4 & 3 & 3 & 4 & $\mathrm{~V}$ & 4 & 4 & 4 & 3 & 3 & 3 & $\mathrm{~V}$ \\
\hline Fraxinus excelsior & 1 & 2 & 1 & 3 & 1 & 1 & $\mathrm{~V}$ & . & . & . & . & . & . & - \\
\hline Fagus sylvatica & . & . & . & + & . & . & $\mathrm{I}$ & . & . & . & . & . & . & - \\
\hline Ulmus glabra & 1 & 1 & 1 & . & . & . & III & . & + & 1 & . & + & . & III \\
\hline Acer campestre & . & . & 1 & . & . & 1 & II & . & + & . & . & 1 & 1 & III \\
\hline Juglans regia & . & . & . & 1 & + & + & III & 1 & 1 & . & . & + & . & III \\
\hline Carpinus betulus & . & . & . & . & + &. & II & . & . & . & + & . & 1 & II \\
\hline Carpinus orientalis & . & . & . & . & . & + & $\mathrm{I}$ & . & . & . & + & + & . & II \\
\hline Morus alba & . &. &. &. & + & + & II & 1 & . &. &. & . & . & $\mathrm{I}$ \\
\hline Cerasus avium & . & + & . & . & + & . & II & . & . & . & . & . & . & - \\
\hline Prunus padus & + & . & . & . & 1 & . & II & . & . & . & . & . & . & - \\
\hline Castanea sativa & . & . & . & . & . & 1 & $\mathrm{I}$ & . & . & . & . & . & . & - \\
\hline Pyrus pyraster & . & . & . & . & . & . & - & . & . & . & 1 & . & . & $\mathrm{I}$ \\
\hline Salix fragilis & . & . & 1 & . & . & . & $\mathrm{I}$ & . & 1 & . & . & . & . & $\mathrm{I}$ \\
\hline Salix alba & . & . & . & . & . &. & - & 1 & . &. & . & . & . & $\mathrm{I}$ \\
\hline \multicolumn{15}{|l|}{ Shrubs layer } \\
\hline Corylus avellana & + & + & . & + & + & + & $\mathrm{V}$ & + & + & . & + & + & + & $\mathrm{V}$ \\
\hline Rubus caesius & 3 & 2 & 2 & 3 & 3 & 3 & $\mathrm{~V}$ & 2 & 3 & 3 & + & 2 & 2 & $\mathrm{~V}$ \\
\hline Crataegus monogy. & . & + & . & . &. & + & II & . & + & . & . & + & . & II \\
\hline Sambucus nigra & . & . & . & . & . & + & $\mathrm{I}$ & . & + & . & + & $\cdot$ & + & III \\
\hline Cornus sanguinea & . & . & . & . & + & . & $\mathrm{I}$ & . & + & . & . & + & . & II \\
\hline Cornus mas & . & . & . & . & . & + & $\mathrm{I}$ & . & . & $\cdot$ & . & + & . & $\mathrm{I}$ \\
\hline Evonymus europae. & . & . & . & . & + & + & II & . & . & . & + & . & . & $\mathrm{I}$ \\
\hline Salix fragilis & . & . & . & . & . & $\cdot$ & - &. & . & + & . & . &. & $\mathrm{I}$ \\
\hline Alnus glutinosa & . & . & . & . & . &. & - & . & . & + & . & . & . & $\mathrm{I}$ \\
\hline Robinia pseudoaca. & . & . & . & + & . & . & $\mathrm{I}$ & . & . & + & . & . & . & $\mathrm{I}$ \\
\hline Morus alba & . & . & . & . & . &. & - & . & . & + & . & . & . & $\mathrm{I}$ \\
\hline Fraxinus excelsior & $\cdot$ & . & . & . & . &. & - & . & + & . & . & . & . & $\mathrm{I}$ \\
\hline \multicolumn{15}{|l|}{$\begin{array}{l}\text { Regeneration } \\
\text { layer }\end{array}$} \\
\hline Fraxinus excelsior & $\cdot$ & + & . & . &. & . & $\mathrm{I}$ & . & + & $\cdot$ & . & + & . & II \\
\hline Acer campestre & . & . & . & . & + & + & II & . & $\cdot$ & $\cdot$ & . & . & . & - \\
\hline Ailanthus altissima & . & . & . & . & $\cdot$ & $\cdot$ & - & $\cdot$ & $\cdot$ & $\cdot$ & . & . & 3 & $\mathrm{I}$ \\
\hline
\end{tabular}


Table 2 (continued): Phytocoenoses with Black Alder (Alnus glutinosa) and Common Ash (Fraxinus excelsior) in the middle and lower stretches of the tributaries (2 = Radimna, 4 Liborajdea, 5 = Berzasca, $6=$ Şirinea, $7=$ Liubotina, $10=$ Mraconia, $11=$ Ogradena, 12 = Eşelniţa).

\begin{tabular}{|c|c|c|c|c|c|c|c|c|c|c|c|c|c|c|}
\hline Lianas & & & . & & & & & & & & & & & \\
\hline Clematis vitalba & . & + & . & . & + & + & III & . & . & + & + & . & . & II \\
\hline Hedera helix & . & + & . & . & + & . & II & . & + & . & . & 3 & . & II \\
\hline Solanum dulcamara & $\cdot$ & + & . & . & . & . & $\mathrm{I}$ & . & . & + & $\cdot$ & . & . & $\mathrm{I}$ \\
\hline Calystegia sepium & . & . & . & . & + & . & $\mathrm{I}$ & . & + & + & . & . & . & II \\
\hline \multicolumn{15}{|l|}{ Tall herb. fringe layer } \\
\hline Petasites hybridus & + & + & . & . & . & . & II & . & . & 2 & . & + & . & II \\
\hline Telekia speciosa & + & + & + & . & . & . & III & . & . & + & . & + & . & II \\
\hline Eupatorium cannabinum & + & $\cdot$ & . & . & . & . & II & . & $\cdot$ & + & · & + & . & II \\
\hline Equisetum telmateija & + & . & . & . & . & . & $\mathrm{I}$ & . & . & + & . & . & . & $\mathrm{I}$ \\
\hline \multicolumn{15}{|l|}{ Herbaceous layer } \\
\hline Parietaria officinalis & 3 & 2 & 2 & + & + & + & $\mathrm{V}$ & 3 & 4 & + & 2 & 3 & 3 & $\mathrm{~V}$ \\
\hline Polygonum hydropiper & + & + & + & + & $\cdot$ & $\cdot$ & IV & + & + & + & $\cdot$ & . & + & IV \\
\hline Mycelis muralis & + & + & + & $\cdot$ & + & . & IV & $\cdot$ & + & . & + & . & • & II \\
\hline Glecoma hederacea & $\cdot$ & + & 3 & + & + & . & IV & + & + & . & $\cdot$ & + & . & III \\
\hline Urtica dioica & + & $\cdot$ & . & + & $\cdot$ & + & III & + & + & + & + & + & + & $\mathrm{V}$ \\
\hline Aegopodium podagraria & $\cdot$ & + & $\cdot$ & $\cdot$ & + & $\cdot$ & II & . & + & + & $\cdot$ & + & + & IV \\
\hline Rumex obtusifolius & $\cdot$ & $\cdot$ & + & + & $\cdot$ & $\cdot$ & II & $\cdot$ & $\cdot$ & + & $\cdot$ & $\cdot$ & • & I \\
\hline Lamium maculatum & + & + & $\cdot$ & $\cdot$ & + & . & III & + & + & . & + & . & . & III \\
\hline Festuca gigantea & $\cdot$ & + & + & $\cdot$ & . & . & II & + & $\cdot$ & $\cdot$ & $\cdot$ & + & + & III \\
\hline Stachys sylvatica & . & + & + & . & + & . & III & + & . & + & . & + & . & III \\
\hline Ranunculus repens & + & + & + & + & $\cdot$ & $\cdot$ & IV & $\cdot$ & + & . & . & $\cdot$ & . & I \\
\hline Brachypodium sylvaticum & + & + & + & $\cdot$ & . & . & III & + & + & . & . & + & . & III \\
\hline Asarum europaeum & + & 2 & . & + & 1 & . & III & . & $\cdot$ & . & . & + & . & I \\
\hline Geum urbanum & $\cdot$ & + & + & + & $\cdot$ & $\cdot$ & III & + & + & . & $\cdot$ & + & + & IV \\
\hline Pulmonaria o. maculosa & $\cdot$ & + & . & . & + & $\cdot$ & II & $\cdot$ & $\cdot$ & . & . & + & . & $\mathrm{I}$ \\
\hline Lathyrus niger & $\cdot$ & + & $\cdot$ & + & $\cdot$ & $\cdot$ & II & $\cdot$ & $\cdot$ & . & . & $\cdot$ & . & - \\
\hline Carex remota & 1 & + & + & + & . & . & IV & + & $\cdot$ & . & $\cdot$ & . & + & II \\
\hline Galeopsis tetrahit & + & $\cdot$ & $\cdot$ & $\cdot$ & . & $\cdot$ & I & + & + & . & . & . & . & II \\
\hline Myosoton aquaticum & $\cdot$ & $\cdot$ & $\cdot$ & . & $\cdot$ & $\cdot$ & - & $\cdot$ & + & 3 & + & . & . & III \\
\hline Stellaria holostea & $\cdot$ & $\cdot$ & $\cdot$ & $\cdot$ & . & $\cdot$ & - & + & $\cdot$ & + & $\cdot$ & $\cdot$ & & II \\
\hline Ajuga reptans & $\cdot$ & $\cdot$ & $\cdot$ & $\cdot$ & . & $\cdot$ & - & + & + & . & . & . & . & II \\
\hline Agrostis stolonifera & $\cdot$ & $\cdot$ & . & . & . & + & $\mathrm{I}$ & $\cdot$ & $\cdot$ & + & $\cdot$ & . & . & $\mathrm{I}$ \\
\hline Bidens tripartita & + & + & $\cdot$ & $\cdot$ & . & $\cdot$ & II & . & . & + & . & . & . & $\mathrm{I}$ \\
\hline
\end{tabular}


Table 2 (continued): Phytocoenoses with Black Alder (Alnus glutinosa) and Common Ash (Fraxinus excelsior) in the middle and lower stretches of the tributaries $(2=$ Radimna, $4=$ Liborajdea, 5 = Berzasca, 6 = Şirinea, 7 = Liubotina, $10=$ Mraconia, $11=$ Ogradena, $12=$ Eșelniţa).

\begin{tabular}{|c|c|c|c|c|c|c|c|c|c|c|c|c|c|c|}
\hline Carex pendula & 3 & . & . & . & . & . & I & $\cdot$ & $\cdot$ & $\cdot$ & . & . & . & - \\
\hline Lysimachia nummularia & 3 & . & . & . & . & . & $\mathrm{I}$ & $\cdot$ & $\cdot$ & . & . & . & $\cdot$ & - \\
\hline Lycopus europaeus & + & + & . & . & . & . & II & . & $\cdot$ & . & . & . & . & - \\
\hline Juncus effusus & + & + & . & . & . & . & II & + & . & . & . & . & $\cdot$ & $\mathrm{I}$ \\
\hline Athyrium filix femina & + & . & + & . & . & + & III & . & + & $\cdot$ & . & . & . & $\mathrm{I}$ \\
\hline Scirpus sylvaticus & . & . & . & + & . & . & $\mathrm{I}$ & . & + & + & . & . & + & III \\
\hline Dryopteris filix mas & . & . & . & . & + & . & I & . & . & . & + & . & + & II \\
\hline Elymus repens & $\cdot$ & . & + & . & . & . & $\mathrm{I}$ & . & . & $\cdot$ & + & . & . & $\mathrm{I}$ \\
\hline Plantago major & . & . & . & . & . & . & I & . & . & . & . & . & + & I \\
\hline Solanum nigrum & + & . &. & . & . & + & II & . & . & $\cdot$ & . & . & . & $\mathrm{I}$ \\
\hline Saponaria officinalis & . & . & . & . & . & . & - & . & . & $\cdot$ & . & . & . & $\mathrm{I}$ \\
\hline Calamintha vulgaris & $\cdot$ & $\cdot$ & $\cdot$ & $\cdot$ & $\cdot$ & $\cdot$ & $\mathrm{I}$ & . & $\cdot$ & + & $\cdot$ & + & . & II \\
\hline Ruscus aculeatus & . & . & . & . & + & . & $\mathrm{I}$ & . & . & $\cdot$ & . & . & . & - \\
\hline Daphne laureola & . & . & . & $\cdot$ & + & . & I & . & $\cdot$ & . & . & . & . & - \\
\hline Asperula taurina & $\cdot$ & $\cdot$ & . & $\cdot$ & + & $\cdot$ & $\mathrm{I}$ & $\cdot$ & $\cdot$ & $\cdot$ & . & . & $\cdot$ & - \\
\hline Tamus communis & . & . & . & + & + & . & II & . & . & . & . & . & & - \\
\hline Arctium minus & $\cdot$ & + & $\cdot$ & $\cdot$ & $\cdot$ & $\cdot$ & $\mathrm{I}$ & + & $\cdot$ & $\cdot$ & . & . & $\cdot$ & $\mathrm{I}$ \\
\hline Stellaria media & . & . & . & + & . & . & I & . & + & + & . & + & . & III \\
\hline Anthriscus sylvestris & . & $\cdot$ & $\cdot$ & + & . & $\cdot$ & $\mathrm{I}$ & . & $\cdot$ & . & . & . & + & I \\
\hline Dipsacus fullonum & + & + & + & . & . & . & III & . & . & . & . & . & + & I \\
\hline Phytolacca americana & $\cdot$ & . & . & $\cdot$ & . & + & $\mathrm{I}$ & . & $\cdot$ & $\cdot$ & + & . & . & $\mathrm{I}$ \\
\hline Chelidonium majus & + & $\cdot$ &. & $\cdot$ & . & + & II & . & $\cdot$ & $\cdot$ & . & . & . & - \\
\hline Tussilago farfara & . & + & $\cdot$ & . & . & + & II & . & $\cdot$ & . & . & . & . & - \\
\hline Erigeron annuus & + & + & + & . & . & . & III & . & . & . & . & . & . & - \\
\hline
\end{tabular}

Species noted with + (I) in one sampling site: 1 - Geranium robertianum, Knautia drymeia, Galium schultesii; 2 - Acer pseudoplatanus reg., Circaea lutetiana, Helleborus odorus, Oxalis acetosella, Polygonatum odoratum, Sambucus ebulus, Verbena officinalis; 3 - Achillea millefolium; 4 - Ailanthus altissima reg., Fagus sylvatica, Aremonia agrimonioides; 5 - Melandrium album, Poa annua; 6 - Physalis alkekengi; 7 - Cichorium intybus, Quercus cerris reg.; 8 - Alnus glutinosa reg., Artemisia annua; 9 - Sisymbrium sophia, Taraxacum officinale; 10 - Humulus lupulus, Lythrum salicaria, Mentha aquatica, Mentha longifolia, Rumex crispus, Salix purpurea, Stachys palustris, Ulmus glabra, Vitis sylvestris, Xanthium strumarium; 11 - Berteroa incana, Echinocloa crus galli, Viola mirabilis; 12 - Artemisia vulgaris, Astragalus glycyphyllos, Centaurea stoebe, Cynodon dactylon, Linaria vulgaris, Salix viminalis, Scabiosa ochrleuca, Scrophularia alata, Setaria viridis, Silene vulgaris, Trifolium repens; 13 - Lunaria rediviva, Salvia glutinosa. 
Place of sampling: 1 (field sample 3) Ieşelniţa Valley: N 4444 18, E 2220 00; 2 (field sample 19) Radimna Valley: N 4449 26, E 2133 37; 3 (field sample 4) Eşelniţa Valley: N 4443 05, E 2221 19; 4 (field sample 27) Ogradena Valley: N 4440 14, E 2218 03; 5 (field sample 25) Ogradena Valley: N 4441 31, E 2220 35; 6 (field sample 10) Şirinia Valley: N 4437 19, E 2201 22, 7 (field sample 9) Liubotina Valley: N 4432 41, E 22 12 31; 8 (field sample 22) Liborajdea Valley: N 4440 43, E 2146 21; 9 (field sample 24) Berzasca Valley: N 4439 50, E 2158 30; 10 (field sample 23) Berzasca Valley: N 4439 10, E 2157 44; 11 (field sample 8) Liubotina Valley: N 44 39 16, E 2212 34; 12 (field sample 5) Mraconia Valley: N 4439 16, E 2216 03; 13 (field sample 6) Mraconia Valley downstream the previous sampling point at $50 \mathrm{~m}$ altitude; 14 (field sample 26) Ogradena Valley: N 4440 35, E 221745.

The riparian gallery like Black Alder forest occurs in the studied area on the tributaries in two different variants (Tab. 2). The first is characterized by high constancy (V) of Black alder and Common ash (Tab. 2, samples column 1-7), in the second the tree layer being edified mostly by Black alder (Tab. 2, samples column 8-14). In both variants the Elm (Ulmus glabra) is present with constancy III. Apart from the tree layer in the Ash-Alder forest (samples column 1-7) the herbaceous layer presents visible differences by the occurrence of some beech forest species of the Fagetalia order like the European ginger (Asarum europaeum), Lungwort (Pulmonaria officinalis ssp. maculosa), Viola reichenbachiana, Black pea (Lathyrus niger), Common spindle tree (Euonymus europaeus) and others.

The regional character of the riparian ash-alder forest is given by some thermophiloussubthermophilous Balcanic and sub-Mediterranean species like Pink woodruff (Asperula taurina), Black bryony (Dioscorea communis), Spurge laurel (Daphne laureola) and Butcher's broom (Ruscus aculeatus, Ruscus hypoglossum). The low constancy was given as a consequence of the exceptional torrential flood in 2014, which washed away a part of the riparian forest herbaceous vegetation. Such situations were stated in Şirinia and Plavişeviţa valleys. The above mentioned species present interest for the area from the phytogeographical point of view (Borza, 1931a, b; Horvat et al., 1974).

The occurrence of Butcher's broom (Ruscus aculeatus) is remarkable as similar occurrences have been observed on the Lower Rhône River in a Mediterranean floodplain forest of White poplar (Populus alba) accompanied by Small leafed ash (Fraxinus angustifolia), Field elm (Ulmus minor) and other species (Grillas and van Wyijck, 1990).

On lower altitudes the Black alder stands are interlocking with willow stands. On the whole the gallery like willow stands with White and Brittle willow (Salix alba, Salix fragilis), as well as White poplar (Populus alba) are poorly represented (Tab. 1, column 4). They occurred on larger scale at the mouth of the tributaries into the Danube and are known as Salici-Populetum Meijer-Drees 1936 (Morariu and Danciu, 1970; Morariu et al., 1973). Due to the backwater situation of the impounded Danube and the loss of river dynamics they mostly disappeared on the lower river stretches being replaced by Magnocaricion and Phragmition reeds characteristic for borders of standing waters.

Apart from the above mentioned habitat types and phytocoenoses, it has to be mentioned as characteristic for the studied riparian habitats a pioneer community edified by Purple willow (Salix purpurea) on open gravel banks (Tab. 1, column 4), locally also with regeneration of Black poplar (Populus nigra), included in the association Saponario-Salicetum 
purpureae (Br.-Bl. 1930) Tschou 1946. These fragmental phytocoenoses are near to those included in the habitat type 3240 Alpine rivers and their ligneous vegetation with Salix elaeagnos. In this habitat type are included as well pioneer gravel and sandy bank habitats with Salix purpurea, as they have been described before the construction of the Iron Gate power plant (Morariu and Danciu, 1970; Morariu et al., 1973; Sanda et al., 2008). Such fragments of Purple willow communities have been observed and studied during our field trips on the lower part of Liborajdea and Plavişeviţa tributaries.

At that time when the Danube and its tributaries were still supposed to natural hydrological dynamics with changes between high and low water levels, on the mouth of the tributaries and as well on the Danube River banks, it developed during low water levels pioneer communities edified by therophyte species constituting phytocoenoses of the association Dichostyli-Gnaphalietum uliginosi (Horvatic 1931) Soó and Timár 1947 (Morariu and Danciu, 1970). As a consequence of the "Iron Gates" Dam construction and the backwater situation on the tributaries pioneer communities are very rare at present. They are developed only fragmental in very small patches, identified for example on the lower Plavisevița Stream and edified by Chenopodium ambrosioides, Portulaca oleracea and Heleochloa alopecuroides.

All the valleys and their rivers and streams are supposed to human activities and interventions. In particular on the lower stretches of the tributary rivers and streams are visible major impacts due to the hydrotehnical constructions and the back water situation created by the accumulation lake and the changes of the hydrological dynamics on the mouth of the tributaries. On these places the riparian vegetation suffered a remarkable shifting from dynamic running waters accompanying vegetation to one of more or less standing waters. Due to this fact also the Wild grape (Vitis sylvestris) which occurred curtain like in softwood riparian galleries (Meusel and Niedermaier, 1985) on the Clisura stretch of the Danube, became continuously rare. In general a large part of the characteristic vegetation of softwood stands disappeared. Impacts are given also through the spreading of alien species, some of them being invasive. Before the construction of the "Iron Gates" Dam was mentioned the association Salici-Populetum (Tx. 1931) MeijerDrees in 1936 with a facies amorphosum fruticosae "on the whole area of the Iron gate, in particular on the island Moldova Veche, occupying large area as well along the Danube River banks” (Morariu and Danciu, 1970; Morariu et al., 1973). On the Danube the species is present on larger scale, also in the back water situation, but on the tributaries Indigo-shrub (Amorpha fruticosa) is scarcely represented. On some of the tributaries (Ogradena, Plavişeviţa) another alien species, the American pokeweed (Phytolacca americana) occurs, but to date not as an invasive species.

The Tree of heaven (Ailanthus altissima), originally from China (Sârbu et al., 2013), is cultivated and used in forest plantations also in the Clisura area, but the species is as well naturalized in riparian habitats, in particular in the Ogradena Valley presenting a large natural regeneration (Tab. 2, column 14). In the management of the Natural Park of the Iron Gate including many habitats of community interest, between them the priority habitat type 91E0, this fact has to be treated with high attention because the Tree of heaven is replacing the natural riparian vegetation.

A considerable impact for the riparian vegetation and the rivers can be stated in the larger valleys of tributaries by misuse of the river banks for diverse waste deposition which enters also the rivers generating water pollution and changing the habitats quality. 


\section{CONCLUSIONS}

The riparian vegetation of all the tributaries have common characteristics, but there are also some distinct differences between the vegetation and habitat types of the studied valleys. These depend on the size of the basin of each of the tributaries, the geomorphological structure of the valley from the springs to the mouth into the Danube, the length of the river, its discharge and its dynamics and as well the changes of the natural conditions by men's intervention like hydraulic measures.

The upper parts of the rivers are in a natural or near natural state, with small alluvial vegetation, the adjacent forests reach the river banks mostly. On the middle and lower stretches the riparian vegetation is well represented by the priority habitat type 91 E0 Alluvial forests with Alnus glutinosa and Fraxinus excelsior with the subtype 44.3: Alno-Padion and 44.13: Salicion albae. The latter suffers on the lower part of the rivers by human impact due to the backwater situation from the Danube water reservoir.

On some of the rivers occur the habitat type 6430 Hydrophilous tall herb fringe communities of plains and of the mountainous to alpine levels being represented with phytocoenoses edified by Petasites hybridus and Telekia speciosa. These characteristic mountainous phytocoenoses attains on the Clisura tributaries area their lowest distribution point in the Carpathians.

Although the riparian vegetation is considered more azonal, it is of interest from the biogeographical point of view, due to some thermophilous and sub-termophilous Mediterranean and sub-Mediterranean species. These species occurs due to the geographical position of the Clisura with influences of a sub-Mediterranean climate. For that reason the riparian vegetation of the South-western part of the country differs from that of other parts of the Carpathians.

\section{ACKNOWLEDGEMENT}

Many thanks to Mr. Berchi M. from WWF-Romania, branch office Reşiţa for his valuable help and assistance during field activities in the Clisura area.

\section{REFERENCES}

1. Borza A., 1931a - Die Vegetation und Flora Rumäniens, Guide de la sixième Excursion Phytogéographique Internationale, Roumanie 1931, Edit. Le Jardin Botanique de l’Université de Cluj, 1-55. (in German)

2. Borza A., 1931b - Botanischer Führer durch die Umgebung von Băile Herculane (Herkulesbad) bis an die Donau, Guide de la sixième Excursion Phytogéographique Internationale, Roumanie 1931, Edit. Le Jardin Botanique de l’Université de Cluj, 56-63. (in German)

3. Borza A. and Boşcaiu N., 1965 - Introducere în studiul covorului vegetal, Edit. Academiei Republicii Socialiste România, Bucureşti, 340. (in Romanian)

4. Ciocârlan V., 2009 - Flora ilustrată a României, Pteridophyta et Spermatophyta, Edit. Ceres, Bucureşti, 1141. (in Romanian)

5. Coste I., 1984 - Contribution à l'étude de l'association Stellario nemori-Alnetum glutinosae (Kästner 1938) Lohm. 1957 en Roumanie, Colloques phytosociologiques, IX, La vegetation des forêts alluviales, Strasbourg 1980, Redacteur: Professeur J.-M. Gehu, J. Cramer in der A. R. Gantner Verlag Kommanditgesellschaft, FL-9490 Vaduz, 65-74. (in French with German and Romanian abstract)

6. Doniţă N. and Roman N., 1976 - Atlasul Republicii Socialiste România, foaia VI-2, Vegetaţia/Vegetation, Unităţi zonale pe altitudine, Edit. Academiei, Bucureşti. (in Romanian and English) 
7. Doniţă N., Popescu A., Paucă-Comănescu M., Mihăilescu S. and Biriş I. A., 2005 - Habitatele din România, Edit. Tehnică Silvică, Bucureşti, 496. (in Romanian)

8. $\quad$ Ellenberg H., Weber H. E., Düll R., Wirth V. and Werner W., 2001 - Zeigerwerte von Pflanzen in Mitteleuropa/Indicator values of plants in Central Europe, Scripta Geobotanica, 18, 1-264, Erich Goeltze K. G., Göttingen. (in German)

9. EUR28, 2013 - Interpretation Manual of the European Union habitats, European Commission DG Environment, 144.

10. Gafta D. and Mountford O. (eds), 2008 - Manual de interpretare a habitatelor Natura 2000 din România, Risoprint, Cluj-Napoca, 101. (in Romanian)

11. Glăvan V. and Geanana M., 1972 - Map X, Solurile, in Atlasul complex "Porţile de Fier", Edit. Academiei Republicii Socialiste România, Bucureşti, 28. (in Romanian)

12. Grillas P. and van Wijck C., 1990 - Le bois de Giraud/Tourtoulen (Arles), Bilan ecologique et propositions pour la gestion de la ripisylve, Station biologique de la Tour du Valat - Le Sambuc, Conservatoire de l'Espace Littoral et des Rivages Lacustres, 52. (in French)

13. Horvat I., Glavac V. and Ellenberg H., 1974 - Vegetation Südosteuropas, Vegetation of SouthEastern Europe, VEB Gustav Fischer Verlag Jena, 768. (in German)

14. Iancu M., 1972 - Valea Carpatică a Dunării, Map IV, in Atlasul complex "Porţile de Fier", Edit. Academiei Republicii Socialiste România, Bucureşti, 22. (in Romanian)

15. Meusel H. and Niedermaier K., 1985 - Die Breitlaubwälder des Süd- und Ostkarpatenraumes in vergleichend chorologischer Sicht, Naturwissenschaftliche Forschungen über Siebenbürgen, III, Beiträge zur Pflanzengeographie des Südost-Karpatenraumes, 1-42, Siebenbürgisches Archiv, 20, Böhlau Verlag Köln Wien. (in German)

16. Mölder A. and Schneider-Binder E., 2011 - On the beautiful diverse Danube? Danubian floodplain forest vegetation and flora under the influence of river eutrophication, River Research and Applications, 27, 881-894, Willey.

17. Morariu I. and Danciu M., 1970 - In der Gegend des Eisernen Tores (Porţile de Fier) gemeldete Pflanzenassoziationen, Revue Roumaine de Biologie, Série de Botanique, 15, 6, 419-429, Editions de l’Académie de la République Socialiste de Roumanie. (in German)

18. Morariu I., Danciu M. and Ularu P., 1973 - Die Vegetation der Flussinsel Moldova Veche, Lucrările Grădinii Botanice din Bucureşti/Acta Botanica Horti Bucurestiensis 1972-1973, 465499, Bucureşti. (in German)

19. Niklfeld H., 1973 - Natürliche Vegetation/Natural vegetation, in Atlas der Donauländer/Atlas of the Danubian countries, Map volume/Map number 171, Distributed on commission by: Franz Deuticke Verlags- und Universitätsbuchhandlung, Wien/Vienna 1970-1989, edited and redacted on behalf of the Federal Ministry of Science and Research and in the name of the Austrian Institute of East and South-East European Studies by Breu J. (in German and English)

20. Posea G., Popescu N. and Grigore M., 1972 - Terasele şi suprafeţele de nivelare, Map VII, in Atlasul complex "Porţile de Fier", Edit. Academiei Republicii Socialiste România, Bucureşti, 25. (in Romanian)

21. Resmeriţă I., Vicol E. C., Boşcaiu N., Coldea G. and Täuber F., 1972 - Vegetaţia din sectorul Ieşelniţa-Tri Kule, in Atlasul complex "Porţile de Fier”, Edit. Academiei Republicii Socialiste România, Bucureşti, 47. (in Romanian)

22. Roman N., 1972 - Vegetaţia din sectorul Orşova - Drobeta - Turnu Severin, in Atlasul complex "Porţile de Fier", Edit. Academiei Republicii Socialiste România, Bucureşti, 46. (in Romanian)

23. Roman N., 1974 - Flora şi vegetaţia din sudul Podişului Mehedinţi, Edit. Academiei Republicii Socialiste România, Bucureşti, 222. (in Romanian)

24. Sanda V., Öllerer K. and Burescu P., 2008 - Fitocenozele din România, Sintaxonomie, structura, dinamica şi evoluţie, Ars Docendi, Universitatea din București, 570. (in Romanian)

25. Sârbu I., Ştefan N. and Oprea A., 2013 - Plante vasculare din România, Determinator ilustrat de teren, Edit. Victor B. Victor, Bucureşti, 1231. (in Romanian) 